\title{
Target-specified and target-categorized conditions in RSVP tasks as reflected by detection time
}

\author{
JUAN BOTELLA \\ Universidad Autónoma de Madrid, Madrid, Spain
}

(Charles W. Eriksen, Sponsor)

\begin{abstract}
An experiment with a rapid serial visual presentation (RSVP) paradigm and target-specified and target-categorized conditions is reported. Among other improvements to the design, a response time measure was included as an index of the time needed to detect the target's key feature. Typical patterns of intrusion were found: posttarget predominance in the target-specified condition and symmetry in the target-categorized condition. Reaction times (RTs) do not show the strictly increasing function predicted from simple parallel models; the shortest RTs were associated with hits and their functions were similar for the two experimental conditions. The results are discussed within the context of parallel views, and an account for the differences between targetspecified and target-categorized conditions is proposed.
\end{abstract}

In a typical rapid serial visual presentation (RSVP) task, a series of stimuli is presented in a single spatial position at a high rate. Subjects have to report some feature of the only item in the series with a prespecified feature. The target defining feature (or key feature) belongs to the key dimension, and the dimension to be reported is designated the response dimension; the value of the target in that dimension is the target's response feature.

In these kinds of experiments, subjects often report response features that are actually presented but that belong to some other item presented near the target. These errors are called intrusions. The most frequent error is the reporting of response features from items presented after the target (posttarget intrusions), most commonly the response feature of the item presented just after the target (intrusion from the +1 position). There are, however, some published experiments in which the pattern found has not been a predominance of posttarget intrusions but a symmetrical pattern of pretarget and posttarget intrusions (McLean, Broadbent, \& Broadbent, 1983, Experiment 2) or even a predominance of pretarget intrusions (Intraub, 1985).

Particularly relevant are experiments with targetspecified and target-categorized conditions (Broadbent \& Broadbent, 1986; Gathercole \& Broadbent, 1984, Experiment 1; McLean et al., 1983, Experiment 2). In a typical experiment with that paradigm (McLean et al., 1983, Experiment 2), the color of the only digit inserted in a

The research reported here was supported by Public Health Research Grant MH-01206, Charles W. Eriksen, Principal Investigator, and by a grant to Juan Botella from the Ministerio de Educacion y Ciencia of Spain. Correspondence may be addressed to Juan Botella, Facultad de Psicologia, Universidad Autónoma de Madrid, 28049 Madrid, Spain (e-mail: juanb@ccuam3.sdi.uam.es). series of letters was to be reported. In the target-specified condition, the subject was informed as to the identity of the target digit before each trial, but in the targetcategorized condition, the subject only knew that the key feature was "any digit" in the series of letters. In all published experiments with that paradigm, a posttarget pattern has been found in the specified condition and a symmetrical pattern, in the categorized condition.

D. E. Broadbent and his colleagues (Broadbent \& Broadbent, 1986; Gathercole \& Broadbent, 1984; McLean et al., 1983) proposed that two modes of processing account for these different patterns of intrusions. They proposed that a posttarget pattern reflects a serial mode of processing of the two relevant dimensions, while a symmetrical pattern reflects a parallel mode. Thus, following their logic, in target-specified conditions the subjects adopt a serial-processing strategy, while in target-categorized conditions they shift to a parallel one.

Several results contradict this logic. Botella and Villar (1989) showed in a Monte Carlo simulation that a simple parallel model can generate the three kinds of patterns (pretarget, symmetrical, and posttarget). Botella and Eriksen (1991) demonstrated that the single processing mode adopted by their subjects (whatever it is) generated symmetrical and posttarget patterns without strategical shifts. Botella and Eriksen (in press) have demonstrated that the response features of pretarget items are available to the subjects, even when a posttarget pattern is observed. In another experiment (Botella, Garcia, \& Barriopedro, 1992), two response dimensions were used: subjects had to report the identity and the color of the only word in uppercase. For word identity pretarget intrusions predominated, and for color posttarget intrusions predominated. If mode of processing is to be inferred from the pattern of intrusions, then in this experiment neither serial nor parallel processing can be inferred (or both of them!). 
The results from target-specified versus target-categorized experiments also pose problems for parallel models (Botella \& Villar, 1989; Gathercole \& Broadbent, 1984; Keele \& Neill, 1978). What has been predicted from a parallel model is just the opposite of the observed result. It has been reasonably assumed that detection of a specific number is faster than the detection of any number. Thus, the average time needed to detect the key feature would be longer for the target-categorized condition. Consequently, the detection will more probably coincide with posttarget items in this condition than in the targetspecified condition. In short, the prediction is a later average position of the response features reported for the categorized condition than for the specified condition. But perhaps the assumption of faster processing of a specified number than of any number is false. The experiment reported below has tried to achieve a more direct measure of detection time for the key feature when the key feature is specified and when it is a category.

To explore the relationships between detection time and feature reported, subjects were required to execute a simple detection response as quickly as possible when the key feature was detected. At the end of the trial, they were presented with a menu from which they chose what they considered to be the correct response feature from a set of alternatives. From a parallel point of view, if trials are classified according to the position of the response feature reported, relative to the target, the order of the average reaction times (RTs) should be

$$
\overline{\mathbf{R T}}_{-2}<\overline{\mathbf{R T}}_{-1}<\overline{\mathbf{R T}}_{0}<\overline{\mathbf{R T}}_{+1}<\overline{\mathbf{R T}}_{+2},
$$

where $\overline{\mathrm{RT}}_{i}$ represents the average $\mathrm{RT}$ of trials in which the response feature of the $i$ item is reported. From this point of view, hits and errors are produced by the same mechanism; hits could be considered as "fortunate" conjunctions.

In the present experiment, classical target-specified and target-categorized conditions are included, but there are some differences in the procedure. First, trials without the key feature were included to detect possible random responses. Second, a response menu, such as was employed by Botella and Eriksen (in press), was used. Third, in the target-categorized condition after each trial, a question was asked about the identity of the number detected, in order to evaluate and eliminate possible false alarms. Fourth, after each trial a subjective rating of the level of confidence of the subjects about their responses was obtained (Intraub, 1985).

\section{METHOD}

\section{Subjects}

Twenty-one undergraduate students from the University of Illinois with normal or corrected-to-normal vision served as paid subjects.

\section{Stimuli and Materials}

The stimuli were presented on a Zenith CRT connected with and controlled by a Zenith Data System computer. Programming was conducted by MEL (Schneider, 1988). Lists of 13 items, 12 letters and one number, were constructed. The number was placed, equiprobably, in positions 6,7 , or 8 of each list. It was drawn equiprobably from the set
1-9. The items were in five colors, blue, green, red, yellow, and white (the computer standard colors), while the background remained always dark gray. For each list, one of the five colors was selected equiprobably for the number. That color was not repeated in the same list. For the four letters of the two positions before and after the number, the other four colors were used, taken randomly without repetition. Once the central set of five items was determined, the colors for the other items were drawn, taking again the colors of the four positions nearest to the target as many times as necessary, with the restriction that two consecutive items never shared the same color. The 12 letters were selected randomly without repetition from the set of English uppercase consonants, although some of them were excluded (the letter $M$ was excluded because it was considered too similar to the $N$ ). "Catch" lists were also constructed, with the only difference being that the number was replaced by another letter not included in the series; they were converted into "no target" lists. Items subtended $0.64^{\circ}$ in height and $0.29^{\circ}$ in width.

\section{Procedure}

The subjects were placed $40 \mathrm{~cm}$ from the screen. Two conditions were included, one target specified and the other target categorized. In the target-specified condition, a number appeared at the beginning of each trial in the center of the screen. The subjects were told that if there is a number included in the series, it would be that number. The subject pressed the spacebar and a cross replaced the number for $500 \mathrm{msec}$ (the fixation point). The cross was replaced by the first item, then by the second, and so on until the last one. The exposure time for each item was $83 \mathrm{msec}$. The subjects had to make a rapid response, as soon as they detected the number, by pressing a key; the RT was recorded at the nearest millisecond. When the series was finished, a question about the color of the number appeared on the screen, along with a menu of six alternatives: one for each of the five colors used and another for "I don't know"; the last one was the correct response in catch trials. Once the response was given, another question appeared on the screen about the subjects' level of confidence in the response, with five alternatives: "sure," "pretty sure," "not sure," "guess," and "I don't know." The last alternative was included for those trials in which subjects responded "I don't know" to the question about the color.

For the target-categorized condition, there were two modifications. The first is that no information was given about the number to be presented. The subjects only knew that it would be the only number in the series. The second modification was that when the presentation of the series of each trial was finished, a question about the identity of the detected number was asked before the question about the color. The subjects responded with the key corresponding to the detected number, or with the 0 key if they had not detected any number (of course, this last alternative was the correct response in catch trials). The subjects were told that the question on the confidence level was about the color response.

In one session of about $1 \mathrm{~h}$, the subjects received three blocks of 65 trials from each condition, with rests of $2 \mathrm{~min}$ between trials and $5 \mathrm{~min}$ between conditions. The target-specified condition was presented first to half of the subjects, and the opposite order was applied to the other half. The first block of each condition was considered as practice and was not used for statistical analysis; the same was true for the first 5 trials of the other blocks. Thus from each subject 120 trials of each condition were collected. Only $10 \%$ of trials on each condition were catch trials.

\section{RESULTS}

The statistical analysis included only those trials of the target-categorized condition in which the correct number was reported (there were $5.7 \%$ of responses with a wrong number in noncatch trials). There were $15 \%$ false alarms in the target-specified condition (color responses on catch trials) and $10.3 \%$ in the target-categorized condition (number and color responses in catch trials). In almost all of these trials with false alarms, the confidence level was in the two lowest ratings (87.5\% in target specified and 
84\% in target categorized). There were also some omissions on the noncatch trials. On $11.8 \%$ of the trials in the target-specified condition no color response was made, and on $18.9 \%$ of the trials in the target-categorized condition no number was reported.

There is a pattern of posttarget predominance in the specified condition and a symmetrical pattern in the categorized condition (Table 1). A sign test was used for the statistical analysis of the intrusion patterns. ${ }^{1}$ The proportion of subjects with an advantage of posttarget over pretarget intrusions is statistically significant in the target-specified condition $(16 / 19, p<.01)$ but not in the target-categorized condition $(11 / 19, p<.20)$. When only the positions adjacent to the target are included, there is again a statistically significant predominance of subjects with more +1 than -1 responses in the target-specified condition (14/18, $p<.05)$ but not in the target-categorized condition $(12 / 18, p<.12)$.

To determine whether there was a significant change in intrusion pattern between conditions, the ratio of posttarget to pretarget intrusions was determined for each subject in each condition. By sign test, ratios were significantly higher in the target-specified condition $(15 / 21$, $p<.05)$.

For each subject, median RT for detecting the key feature was calculated for each set of trials, grouped according to the position of the reported color (the analysis of the RTs does not include all subjects because there were some subjects without sufficient trials in some cells). A three-way ANOVA was performed on those values (subjects $\times$ conditions $\times$ positions). The effect for position was significant $[F(4,64)=5.63, p<.0001]$ but there was no significant effect for condition $[F(1,16)=3.18$, $p<.10]$ or for the interaction between position and condition $(F<1)$. Table 2 shows the mean of the median RTs as a function of the experimental condition and of the position of the response feature reported.

The average detection RT for trials according to the confidence level was also calculated, and an ANOVA was performed on those data (subjects $\times$ conditions $\times$ confidence level). The effect for confidence level was significant $[F(3,51)=10.21, p<.001]$, but there was no significant effect for condition $[F(1,17)=1.49, p<.25]$ or for

Table 1

Percentages of Responses for Which the Response Feature Reported Comes From Each of the Positions in the Central Set

\begin{tabular}{lccccc}
\hline & -2 & -1 & $T$ & +1 & +2 \\
\hline Specified & 5.80 & 11.50 & 58.02 & 18.42 & 6.26 \\
Categorized & 8.38 & 14.78 & 52.18 & 17.57 & 7.10 \\
\hline
\end{tabular}

Table 2

Mean of the Median Detection RTs as a Function of the Experimental Condition and of the Position of the Response Feature Reported

\begin{tabular}{lccccc}
\hline & -2 & -1 & T & +1 & +2 \\
\hline Specified & 379 & 387 & 374 & 396 & 411 \\
Categorized & 397 & 407 & 391 & 414 & 420 \\
\hline
\end{tabular}

Table 3

Average Levels of Confidence for Responses, According to the Experimental Condition and to the Pocition of the Response Feature Reported

\begin{tabular}{lccccc}
\hline & -2 & -1 & $T$ & +1 & +2 \\
\hline Specified & 2.13 & 2.47 & 3.20 & 2.50 & 1.97 \\
Categorized & 2.46 & 2.52 & 3.02 & 2.50 & 2.25 \\
\hline
\end{tabular}

the interaction between confidence level and condition $(F<1)$; the average RTs increased as the confidence level decreased (RT means were 391, 394, 418, and 423, respectively). To have a value representing the average level of confidence of the responses for each position, values 1 , 2,3 , and 4 were associated, respectively, to the "guess," "not sure," "pretty sure," and "sure" responses. Table 3 shows the average medians calculated over those values.

\section{DISCUSSION}

The patterns of intrusion errors in this experiment are essentially the same as reported previously, that is, reliably posttarget in the targetspecified condition and symmetrical in the target-categorized condition. The general conclusions for both of the experimental conditions are that the shorter the detection times (1) the more probable are hits, (2) the earlier are the average positions of the reported response features, and (3) the more confident are subjects about their responses.

In many aspects, the relationship between detection time and response position is as expected from a parallel model. A first important point is that the difference between the target-specified and target-categorized conditions seems to be quantitative, not qualitative, at least as reflected by the RT data. The two functions suggested by the data in Table 2 are similar; there is no interaction between the conditions and the RTs associated with the positions of the reported features. The general difference between the conditions does not reach statistical significance, despite being quite stable for the different positions. The relationship observed for errors is basically as expected. RTs are longer when postintrusion errors occur than when the errors are preintrusion. However, there is a departure from this pattern for the RTs associated with hits.

Although prior research (Dick, 1971; Posner, 1970) suggests that detecting any number would be slower than detecting a particular number, the present experiment has not found significant differences in detection RT between the two experimental conditions. Perhaps the different outcomes (if indeed they are different) may result from the difference in response requirements between the earlier research and the present study. As contrasted with the earlier research, subjects in the present experiment had essentially a dual task. They had not only to detect but to determine the color of the target. Let us suppose that processing of the relevant dimensions needs some attentional resources. If more attentional resources are devoted to the key dimension in the categorized condition, fewer resources are available to the response dimension. In such a case, longer time intervals are needed for the output from the responsedimentsion analysis to become available. As a consequence, it is more probable that detection coincides with outputs from earlier items. As the different allocation of resources can partially compensate for the differential difficulty associated with the key dimensions, the difference in detection RT is not significant in the RSVP task.

The main departure of these results from the predictions of parallel models is that the shortest detection RT is that associated with correct responses. To accommodate this result, the model needs some modifications. A speculative but perhaps useful idea could be the following. The performance of the nervous system fluctuates, with moments of higher and lower efficiency. Probably there is a proportion of trials in which the subjects have enough time, while the target is present, to build up a complete and integrated percept, with a clear image of the full stimulus. In these trials, the system works in a very efficient way: the detection time is short, the color response is correct, and the subjects feel very confident with their responses. The easier the experimental condi- 
tion, the more probable that a given level of activation would produce a trial of this kind. There is another set of trials performed in a much less efficient way, with longer RTs, subject to inappropriate recombinations of features and with a lower level of confidence. Performance in the second kind of trials may fit a parallel model like the one described by Botella and Villar (1989). Of course, these two modes represent the extreme points of a continuum in the level of efficiency, and that level changes from moment to moment. A mixture of trials performed in these two stereotyped modes could produce the data of Table 2 and the patterns of Table 1.

\section{REFERENCES}

Botella, J., \& ErIKSEN, C. W. (1991). Pattern changes in rapid serial visual presentation tasks without strategic shifts. Bulletin of the Psychonomic Society, 29, 105-108.

Botella, J., \& ERIKSEN, C. W. (in press). Filtering versus parallel processing in RSVP tasks. Perception \& Psychophysics, 51, 334-343.

Botella, J., GARCIA, M. L., \& \& BARRIOPEDRo, M. I. (1992). Intrusion patterns in rapid serial visual presentation tasks with two response dimensions. Manuscript submitted for publication.

Botella, J., \& Villar, M. V. (1989). Modelos de procesamiento en tareas de presentacion de series visuales. Psicologica, 10, 179-203.

Brondbent, D. E., \& Brondbent, M. H. P. (1986). Encoding speed of visual features and the occurrence of illusory conjunctions. Perception, 15, 515-524.

Dick, A. O. (1971). Processing time for naming and categorization of letters and numbers. Perception \& Psychophysics, 9, 350-352.
Gathercole, S. E., \& Brondbent, D. E. (1984). Combining attributes in specified and categorized target search: Further evidence for strategic differences. Memory \& Cognition, 12, 329-337.

INTRAUB, H. (1985). Visual dissociation: An illusory conjunction of pictures and forms. Journal of Experimental Psychology: Human Perception \& Performance, 11, 431-442.

KeELe, S. W., NeILl, W. T. (1978). Mechanisms of attention. In E. C. Carterette \& P. Friedman (Eds.), Handbook of perception (Vol. 9, pp. 3-47). New York: Academic Press.

McLean, J. P., Broadbent, D. E., \& Broadbent, M. H. P. (1983). Combining attributes in rapid sequential visual presentation tasks. Quarterly Journal of Experimental Psychology, 35A, 171-186.

PosNER, M. I. (1970). On the relationship between letter names and superordinate categories. Quarterly Journal of Psychology, 22, 279-287.

SCHNEIDER, W. (1988). Micro Experimental Laboratory: An integrated system for IBM PC compatibles. Behavior Research Methods, Instruments, \& Computers, 20, 206-217.

\section{NOTE}

1. The number of comparisons made does not always equal the total number of subjects (21) because there were some ties; ties were excluded from sign-test analysis.

(Manuscript received November 4, 1991.) 